\title{
Effect of Placental Extract on Omentum Mesenchymal Stem Cells Differentiation in NMRI Mice
}

\author{
Maryam Sadat Nezhadfazel ${ }^{1}$, Kazem Parivar ${ }^{1}$, Nasim Hayati Roodbari ${ }^{1}$ \& Mitra Heydari Nasrabadi ${ }^{2}$ \\ ${ }^{1}$ Department of Biology, Science and Research Branch, Islamic Azad University, Tehran, Iran \\ 2 Department of Biology, Parand Branch, Islamic Azad University, Parand, Iran \\ Correspondence: Kazem Parivar. Department of Biology, Science and Research Branch, Islamic Azad \\ University, Tehran, Iran. Email: kazem_parivar@yahoo.com
}

Received: July 31, 2017

Accepted: August 23, $2017 \quad$ Online Published: November 10, 2017

doi:10.5539/jmbr.v7n1p176

URL: https://doi.org/10.5539/jmbr.v7n1p176

\begin{abstract}
Omentum mesenchymal stem cells (OMSCs) could be induced to differentiate into cell varieties under certain conditions. We studied differentiation of OMSCs induced by using placenta extract in NMRI mice. Mesenchymal stem cells (MSCs) were isolated from omentum and cultured with mice placenta extract. MSCs, were assessed after three passages by flow cytometry for CD90, CD44, CD73, CD105, CD34 markers and were recognized their ability to differentiate into bone and fat cell lines. Placenta extract dose was determined with IC50 test then OMSCs were cultured in DMEM and 20\% placenta extract.The cell cycle was checked. OMSCs were assayed on 21 days after culture and differentiated cells were determined by flow cytometry and again processed for flow cytometry. CD90, CD44, CD73, CD105 markers were not expressed, only CD34 was their marker. OMSCs were morphologically observed. Differentiated cells are similar to the endothelial cells. Therefore, to identify differentiated cells, CD31 and FLK1 expression were measured. This was confirmed by its expression. G1 phase of the cell cycle shows that OMSCs compared to the control group, were in the differentiation phase. The reason for the differentiation of MSCs into endothelial cells was the sign of presence of VEGF factor in the medium too high value of as a VEGF secreting source.
\end{abstract}

Keywords: OMSCs, CD Markers, Placenta Extract, Cell Cycle, Differentiation

\section{Introduction}

Stem cells have remarkable potential to develop into many different specialized cells in the body. Serving as a sort of repair system for the body, they can theoretically divide without limit to repletion other cells as long as the person or animal is still alive. When a stem cell divides, each new cell has the potential to either endure a stem cell or become another type of cell with a more specialized function, such as muscle cell, red blood cell, or brain cell. MSCs are a group of cells present in bone-marrow stroma and the stroma of various organs with the capacity for mesoderm-like cell differentiation into, many types like osteoblasts, adipocytes, and chondrocytes (Prakash, 2009). Adult stem cells (ASCs), are able to divide into a variety of different cells. The result is of utmost importance in stem cell researches. ASCs with stem cell properties have previously been identified in the bone marrow mesenchyme, skin, hair, dental pulp, kidney, and even peripheral blood (Karaoz et al., 2009). However, adult cells with stem cell properties are safer and have greater practical use, having been employed for over 50 years to replace bone marrow in leukemia and non- hematological diseases without causing malignant transformation (Klopp et al., 2012).During the last few year's isolations of AMSCs from different sources have been reported. It has also been shown promising potential of omental cells for proliferation and differentiation into different cell types (Witowski \& Jorres, 2006). MSCs also are activated and respond to stress or injury. When supplied exogenously, MSCs home to sites of injury primarily inflamed or broken blood vessels. These products can promote angiogenesis, regeneration, remodeling, immune cell activation or suppression, and cellular recruitment (Amy et al., 213). Omentum is considered as a source of ASCs and the non-fatstromal cells in the expanded omental tissue. The omentum is a sheet-like tissue attached to the greater curvature of the stomach and contains secondary lymphnodes called milky spots .Omental mesenchymal stem cells (OMSCs), however, are more easily obtainable in large quantities, can be harvested from the patient's own omentum, and able to passage in culture and differentiates into target tissues (Dhanasekaran et al., 213; Klopp et al., 2012). Together, the data suggest that the omentum contains the potent ability of tissue regeneration and may be useful for treatment of various types of 
diseases involving tissue damages. Many scientists tried to obtain differentiation of ASCs by using some inducers. For example they used growth factors, testicular conditioned medium, follicular fluid, gonadotropins and retinoic acid for various types of stem cells (Dyce \& Li, 2014; Nikoozad et al., 2014). In this work, we used placental extract on OMSCs, induce them by placental growth factors (Miyamoto et al., 2004; Zhang, 2004). Over the past 10 year, various studies have shed light on several important aspects of placenta. The placenta is generally recognized for important functions such as nutrition, respiration and excretion as well as maintenance of fetomaternal tolerance. The components of this organ include the fetal membranes, umbilical cord and trophoblast (Parolini et al., 2009). We have already mentioned a number of physiological aspects of the placenta, but the placenta contains not only the three major nutrient groups: carbohydrates, proteins, and fats, but also a wide range of nutrients such as minerals, vitamins, enzymes, and nucleic acids. Also you could call placental growth factors which switch on the process of cell division. It is important to note that even tiny quantities demonstrate this power. Main Growth Factors of the Placenta included the following (Kentaro, 2001). Hepatocyte Growth Factor (HGF), Nerve Growth Factor (NGF), Epidermal Growth Factor (EGF), Fibroblast Growth Factor (FGF): Promotes growth of fibroblasts, glia cells, and vascular endothelial cells, Insulin-like Growth Factor (IGF), Colony-Stimulating Factor (CSF), Interleukin-1 (IL-1): (T-cells, B-cells, and NK-cells),Interleukin-2 (IL-2): Promotes growth of T-cells. Interleukin-3 (IL-3), Interleukin-4 (IL-4): Promotes growth of B-cells, and promotes division of antibody-producing cells. Protein PLGF (Placental Growth Factor) or placental growth factor is also one of the most important proteins in placental stimulating angiogenesis that is expressed in trophoblast eyelashes and endothelial cells, such as Umblical Vein Endothelial Cell during the embryonic period, which are results formation of blood vessels in the placenta (Athanassiades, 1998). Placenta growth factor (PLGF) is a homodimeric glycoprotein, belonging to the vascular endothelial growth factor (VEGF) sub-family. PLGF in other tissues, including the heart, lungs, muscles, thyroid and fat tissue in the endothelial cells, have been identified. PLGF expressed in Trophblast, monocytes and erythroid cells have also been reported. Factor in tumor angiogenesis are considered strong as one of the factors (Hoeben et al., 2004). MSCs are being used for the treatment of a variety of clinical conditions. Cell-based therapies are quickly taking hold as a revolutionary new approach to treat many human diseases. Among the cells used for therapeutic functions, multipotentmesenchymalstem cells, also often and imprecisely termed MSCs, are widely used because they are considered clinically safe, unique in their immune-capabilities, easily obtained from adult tissues, and easily developed and restored (Darvin et al., 2013). The MSCs expression of cell surface markers including positive for the CD90, CD105 and CD73 markers and negative for CD34 (Teresa et al., 2016; Dominici et al., 2006). These properties led to development of progressive methods to isolate and characterize MSCs from various therapeutic applications in regenerative medicine. In present study, we have isolated MSC cells from omentum, and placed them in the vicinity of placenta extract. We also expressed the specific cell surface antigens using flow cytometry in 21 day of cell differentiation.

\section{Method and Methods}

\subsection{Isolation of OMSCS}

OMSCs Cell isolation was performed according to Chen and his colleagues, with some modifications as described below (Chen KS, Chen, 2012) Omentum (1 mm2 segments) was isolated from neonatal NMRI mice (Pasteur Institute of Iran) aged 12 days and weighting 15-16 gthrough abdominal surgery, washed with phosphate-buffered saline (PBS) to remove any contamination and incubated with a solution containing $2 \mathrm{ml}$ trypsin $(0.25 \%$, Gibco, UK) for $15 \mathrm{~min}$ at $37{ }^{\circ} \mathrm{C}$. After incubation, the cell suspensions were centrifuged at $1500 \mathrm{~g}$ for $5 \mathrm{~min}$ at $4{ }^{\circ} \mathrm{C}$ and tissue pellet was transferred to a new flask for the next round of digestion. The procedure was repeated for 15,20 and $40 \mathrm{~min}$.

Culture of OMSCs: After centrifugation, the cell pellet was washed with Dulbecco's Modified Eagle's Medium (DMEM,Gibco, UK) containing 10\% fetal bovine serum (FBS, Gibco, UK) and 1\% penicillin/ streptomycin(Pen/ Str, Sigma, USA), resuspended in the same medium and plated in a $25 \mathrm{~cm} 2$ cell culture flask (Jet/ Biofil, Italy). Non- adherent cells were removed 3 days later by two time's brief washing with medium. The dishes were incubated in humidified atmosphere of $95 \%$ air and $5 \% \mathrm{CO} 2$ at $37^{\circ} \mathrm{C}$, and the medium was changed every three days. When cultures reached the optimum confluence, cells were lifted by incubation with PBS containing trypsin $(0.25 \%)$ and $0.02 \%$ EDTA at $37{ }^{\circ} \mathrm{C}$. Detached cells were suspended, washed in DMEM, centrifuged at $1500 \mathrm{~g}$ for $5 \mathrm{~min}$ and seeded on three new $25 \mathrm{~cm} 2$ cell culture flasks (ratio 1:3). Anequal volume of stromal medium was added to in activate the trypsin and the suspension was transferred to a conical centrifuge tube. Afte rcentrifuging at $1500 \mathrm{~g}$ for $5 \mathrm{~min}$, the cell pellet was suspended again in 1 to $2 \mathrm{ml}$ of cryopreservation medium (the same formulation as that used topropagate the cells with $20 \%$ FBS, $10 \%$ dimethyl sulfoxide (DMSO, Sigma, USA) and transferred into a liquid nitrogen container for long-term storage (Bunnell et al., 2008). After three passages OMSCs (Figure 1) were characterized by flow cytometric method. 


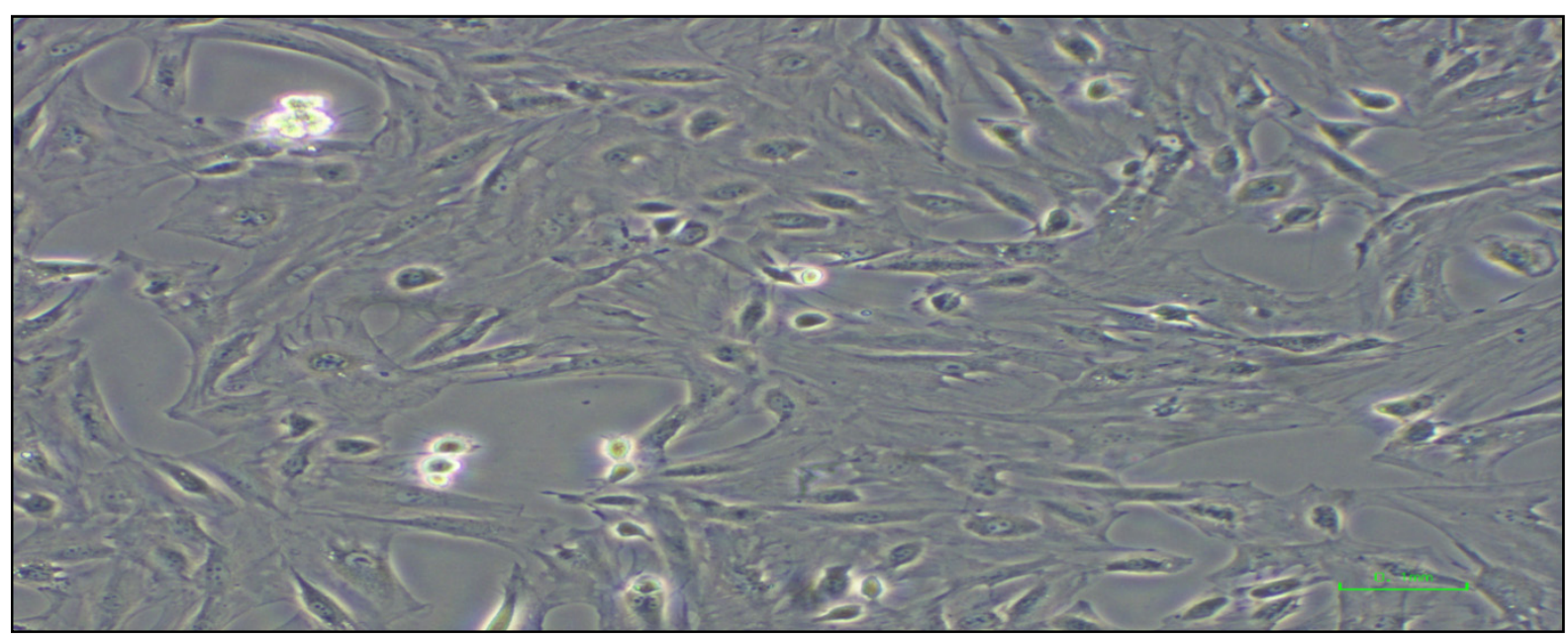

Figure 1. Inverted microscopic image of adherent omentum mesenchymal stem cells after 2 weeks. (With a scale bar of $0.1 \mathrm{~mm}$ )

\subsection{Preparation of Extracts from Placenta}

The following procedure involves handling fresh tissue and there is a serious biohazard risk. Wear latex gloves and full length lab coat. Be exceedingly careful during those steps where sharp implements are used (scissors for mincing tissue, etc.).

\subsection{Procedure}

1) Obtain one fresh full-term mice placenta within 30 minutes of delivery. Drain excess blood, place placenta in red plastic tissue bag (provided at Delivery room), and then pack in ice in igloo cooler for transport back to lab.

2) Placenta was separated from connective tissues and umbilical cordand.

3) So take pieces of placenta and place them in a beaker on ice. All subsequent steps are done at $4^{\circ} \mathrm{C}$.At intervals, use scissors to finely mince tissue in beaker and place it in a large plastic centrifuge bottleon ice which contains ice-cold 1 X PBS buffer (include $100 \mu \mathrm{g} / \mathrm{mL}$ streptomycin and $100 \mathrm{U} / \mathrm{mL}$ penicillin). when all tissuesare minced, swirl in bottle in 1 XPBS buffer (include $50 \mathrm{mM}$ Tris- $\mathrm{HCl}, 150 \mathrm{mM} \mathrm{NaCl}, 150 \mathrm{mM}$ sucrose, $\mathrm{pH}$ 7.2) for $1 \mathrm{~min}$ to wash out blood cells.

4) Place fresh tissue in Waring Blender in cold room with an equal volume Buffer (DMEM high glucose, sucrose, $2 \mathrm{mM}$ EDTA) to Homogenation. Homogenize for amplitude 60 , pulse $4,45 \mathrm{~s}$ and repeat 8 . So centrifuge (170 $\mathrm{g}$ for 15 min at $4^{\circ} \mathrm{C}$ ) and finally Freeze inaliquots in liquid N2 and store at $-80^{\circ} \mathrm{C}$ (Katzman \& Doisy, 1932).

\subsection{Determination of IC50}

In this research, the msenchymal stem cells were affected by placenta extract. At first the IC50 dosage should be identified. The IC50 is abbreviation of 50\% inhibitory concentration that assay by Trypan Blue Exclusion and MTT tests (Sebaugh, 2011). After establishing and sticking to the bottom of the flasks, MSCs treatment with placenta extract and the medium was replaced by fresh medium and placenta extract for up to 21 days in every 3 days. MSCs were treated, every week, and they were observed for any changes by inverted microscope. On the 21 st day, flow cytometry was performed again to make sure that mesenchymal markers of differentiation has occurred. After this point, we started the cell detection.

\section{Results}

After three passages OSCs were characterized by flow cytometric method. They Showed possitive expression for CD73-PE, CD90-FITC, CD105-FITC and CD44-FITC negative Expression for CD34-FITC and CD45-FITC (Figure 2). Also the point graph for OMSCs populations were examined it showed that cells using flow cytometry, we determined the purity of the cells in the obtained isolated cells and found that the purity of the cells ranged were $86.7 \%$ (Figure 3). 

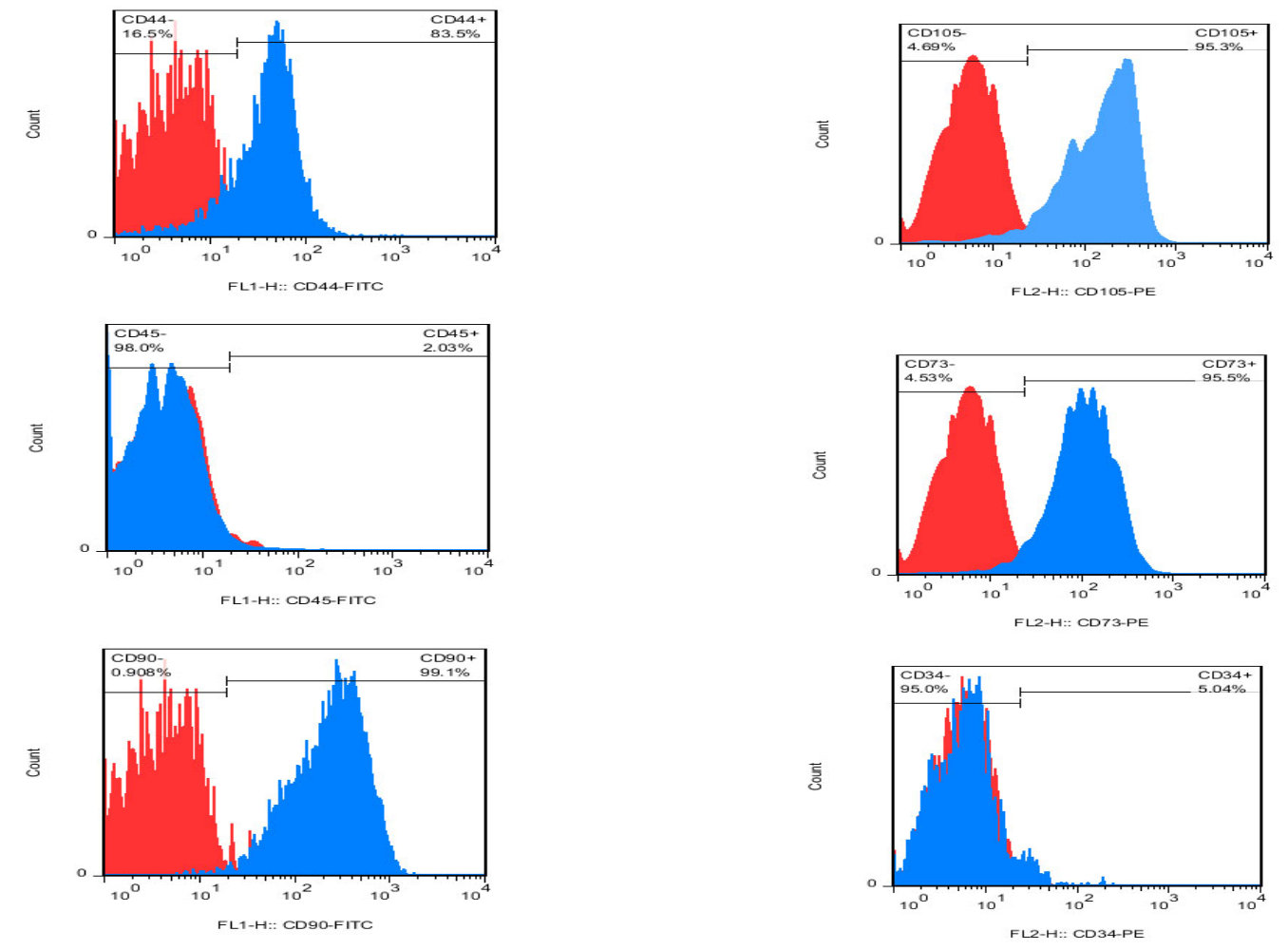

Figure 2. Flow cytometric histograms, expression of CD90, CD45, CD44, CD73, CD105, CD34 markers. Flow cytometry analysis showing that OMSCs expressed MSC surface markers CD44, CD73, CD90 and CD105, but did not express CD34, and CD45

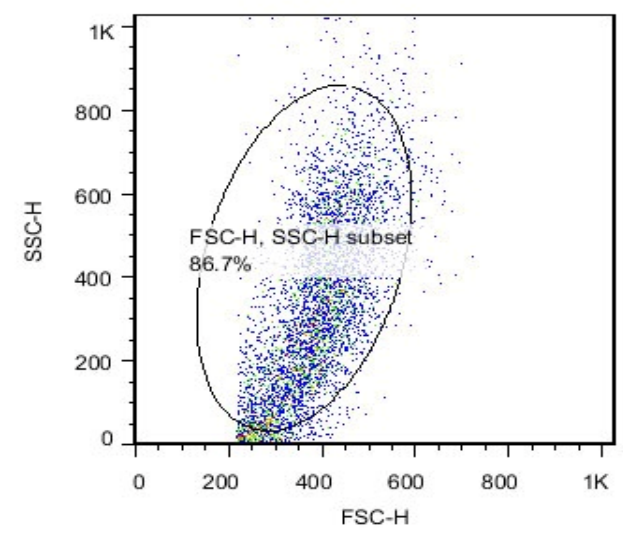

Figure 3. Flow cytometry analysis. Dot plot in arbitrary units on a linear scale showing FSC (forward scatter) and SSCH (side scatter). Forward and side scatter data can be used to classify samples by size (FSC) and by internal complexity (SSC). The characteristic position of omentom stem cells populations were determined by cell size and granularity. The purity of the cells in the obtained isolated cells and found that the purity of the cells ranged ranged were $86.7 \%$

\subsection{Tests the Ability of OMSCs Differentiation}

Next, tests were ability to cell differentiation. Passage 3 used to differentiate cells and can differentiate into cell lines examined the bones and fat.

1) Differentiation into bone: After the plates were covered with cells, environment with a medium inducing differentiation to bone which includes M199environment, containing \%10FBS, 50 micrograms per ml 
Ascorbic2-Phosphate(Sigma), $10 \mathrm{nM}$ and $10 \mathrm{mM} \beta$-Glycerol Phosphate Dexametasone(Sigma), was replaced. As a control group, some houses with the normal DMEM media were stored, which contain $10 \%$ of FBS. The plates were cultured, for 21 days at $37^{\circ} \mathrm{C}$ and $\mathrm{CO} 2$ incubation. Every two days, the medium is replaced. At the end of differentiation time, the cells were staining with Alizarin red (Zhang et al., 2012) (Figure4).

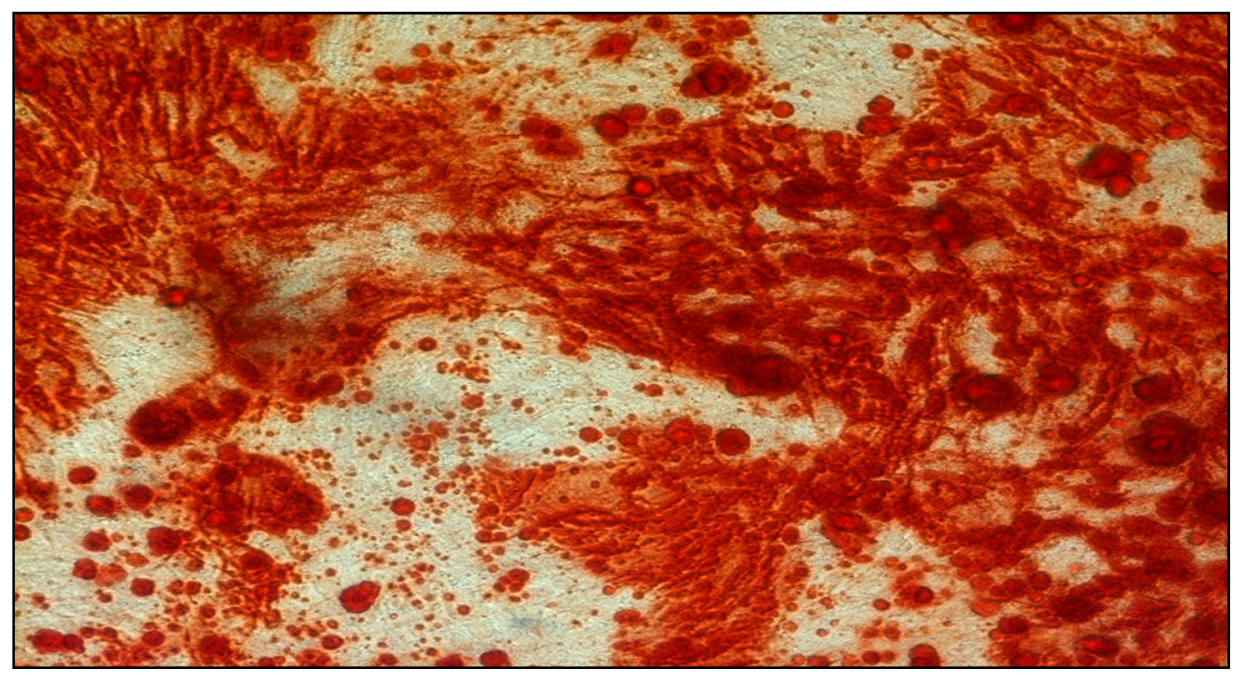

Figure 4. In vitro osteogenic differentiation from OMSCs. Osteogenic differentiation evidenced by morphology of cells after 21 days of culturing in induced medium. Formation of mineralized matrix shown by Alizarin red (Magnification, $\times 400$ )

2) Differentiation into fat for this purpose, the cells were used from passaged 3. The cells were cultured at plate with six wells. When the floor was covered by monolayer cells, the normal culture medium for differentiation into fat substitute was common. Medium, DMEM medium containing 10\% FBS, $100 \mathrm{nM}$ Dexamethasone and Indomethacin (Sigma) were 50 micrograms per milliliter. The control group was considered. Cell cultures for 21 days at $37^{\circ} \mathrm{C}$ and $\mathrm{CO} 2$ incubation and every two days, the fresh medium was replaced. After 21 days the cells were staining by Oil red (Zhang et al., 2012) (Figure 5).

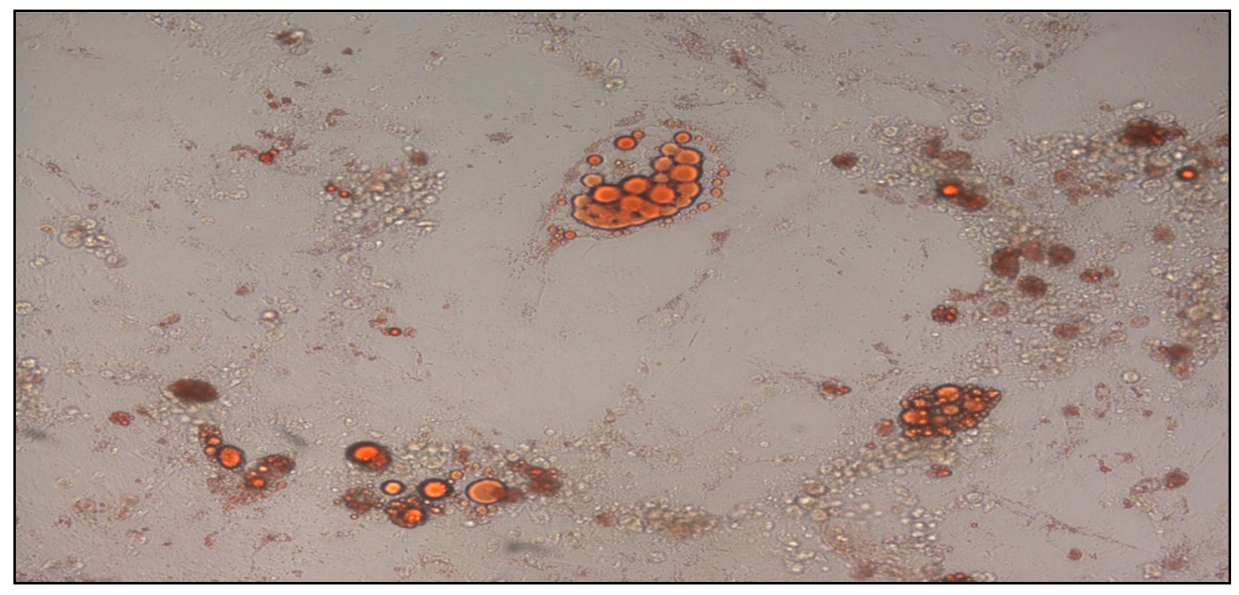

Figure 5. In vitro adipogenic differentiation from OMSCs. Omentum mesenchymal stem cells, were induced with adipogenic differentiation medium. The cells after 21 days of culturing in an induced medium, were staining with Oil red (magnification, $\times 400$ )

(IC50\%) of placental extract dose on OMSCs was 20\%. So MSCs placed in the vicinity of these value of placental extract (Figure 6). 


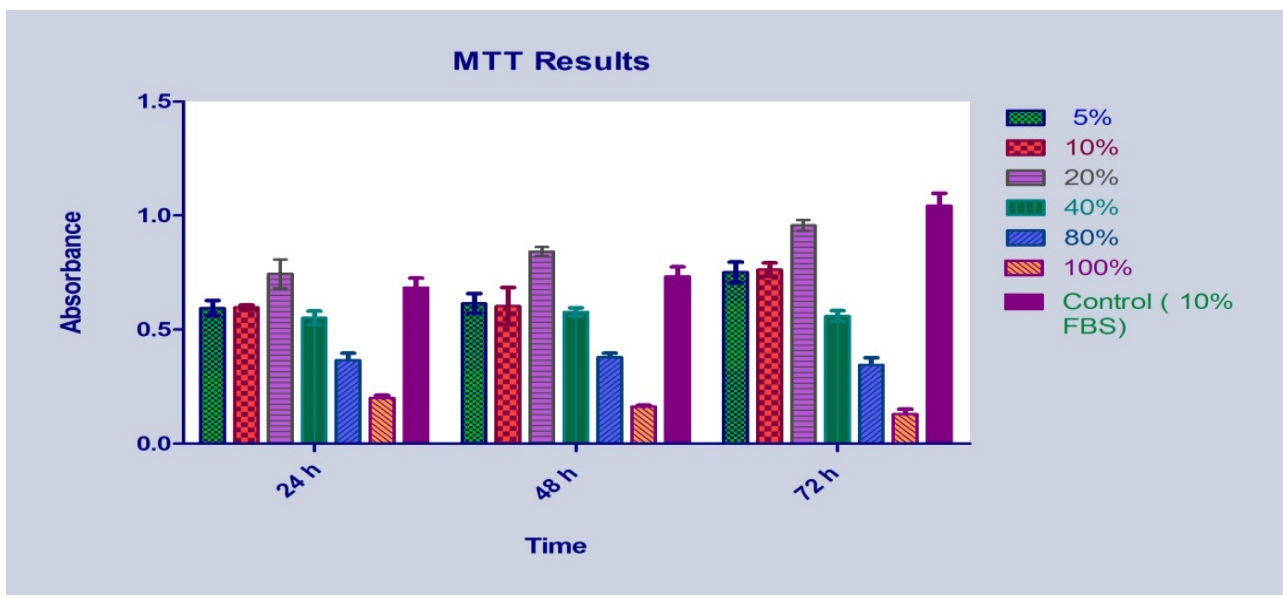

Figure 6. Cell viability measured by MTT assay for placental extract. It shows OMSCs which were treated with different concentration of placental extract for 24,48 and $72 \mathrm{hrs}$. Graph is \% cell viability against concentration of placental extract. From the above graph, IC50 for paclitaxel was $20 \%$

MSCs were established and sticked to the bottom of the flasks, the medium was replace every 3 days with $20 \%$ placenta extract for 21 days. Every week, they were observed for any changes in the appearance by inverted microscope. On the 21st day, the first step was to know that differentiation of MSCs were occurred or not. So any morphological changes examined. Some cells were slightly shorter and seemed wider than others (Figur 7).

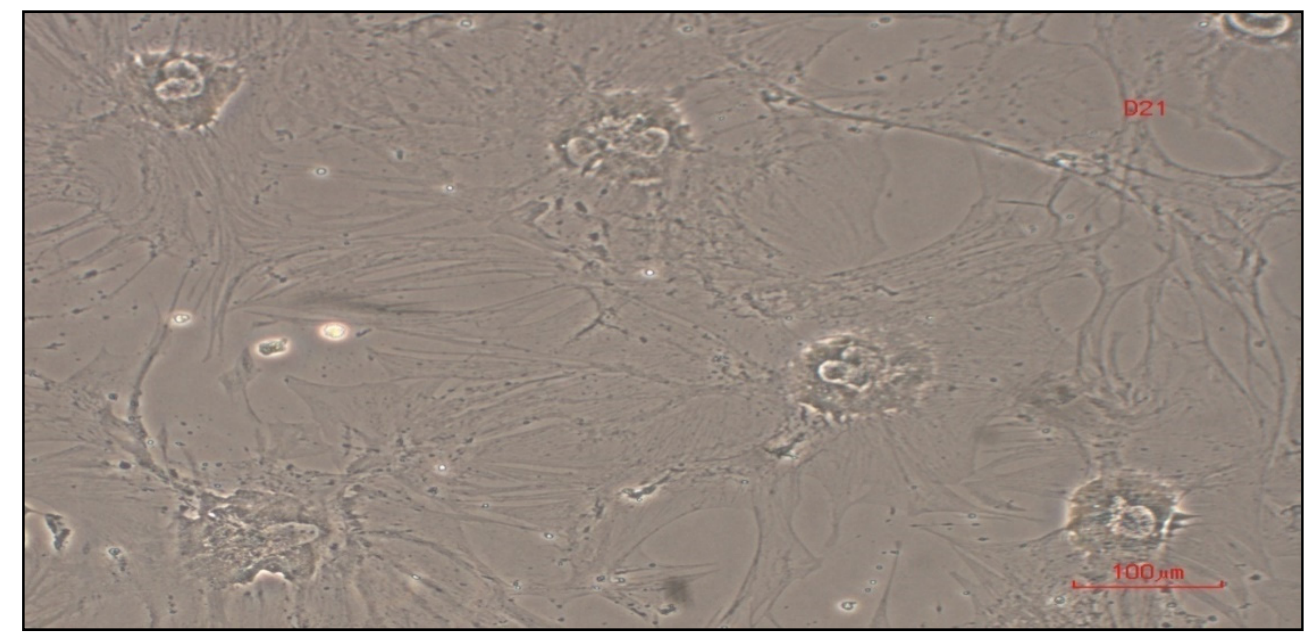

Figure 7. Morphology of OMSCs, after 21st day. Microscopic view of OMSCs which differentiated to endothelial cells. (With a scale bar of $100 \mu \mathrm{m}$ )

During development from stem to fully differentiated, cells in the body alternately divide (mitosis) and appear to be resting (interphase). This sequence of activities exhibited by cells are called the cell cycle. The cell cycle consists of four distinct phases: G1 (Gap1) phase, S phase (synthesis), G2 (Gap2) phase (known as interphase) and M phase (mitosis) (Phil et al., 2011). G2/M phase of cell cycle is a period of rapid cell growth, that protein synthesis and cell preparation for differentiation was revealed in normal cell cycle control sample. This means that all stages of the cell cycle was performed and the cells were growing and multiplying. But in the experimental example, when MSCs were in the presence of placenta extract, G2 / M phases don't have expressed as well as the synthesis or S phase was also significantly dropped. But the G1 phase remains to be seen even more than a control chart. Increased expression of this phase of the cell cycle, suggesting that cell proliferation is inferior, and now the cells have gone more towards differentiation (Figure 8). G1 phase represents the differentiation status of stem cells (Ashley et al., 2012). 

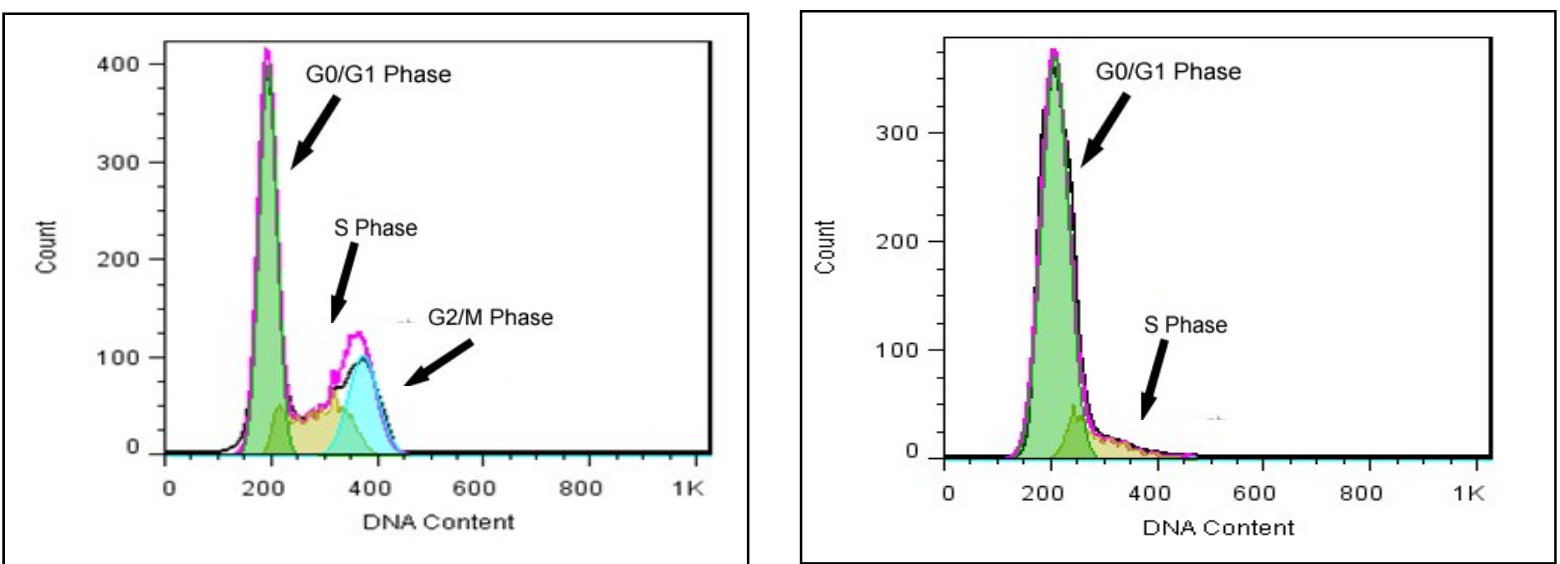

Figure 8. Left histogram: control MSCs cycle and right graph: The MSCs treated by placenta extract cell cycle shows. The next steps were an expression of the markers CD73, CD90, CD105, CD34 and are being re-evaluated (Figure9)

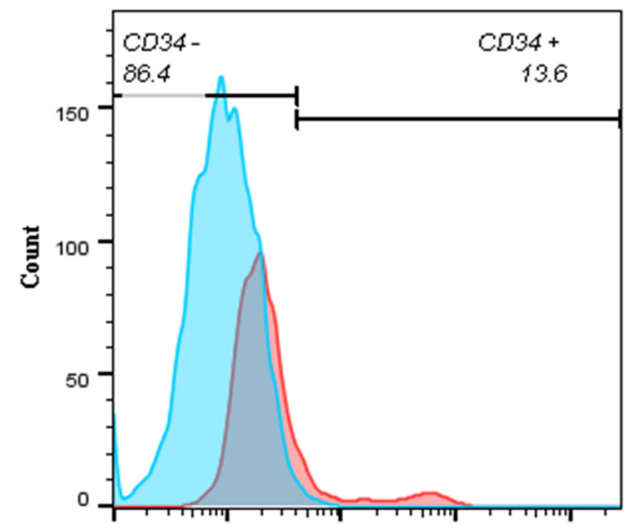

CD34FITC

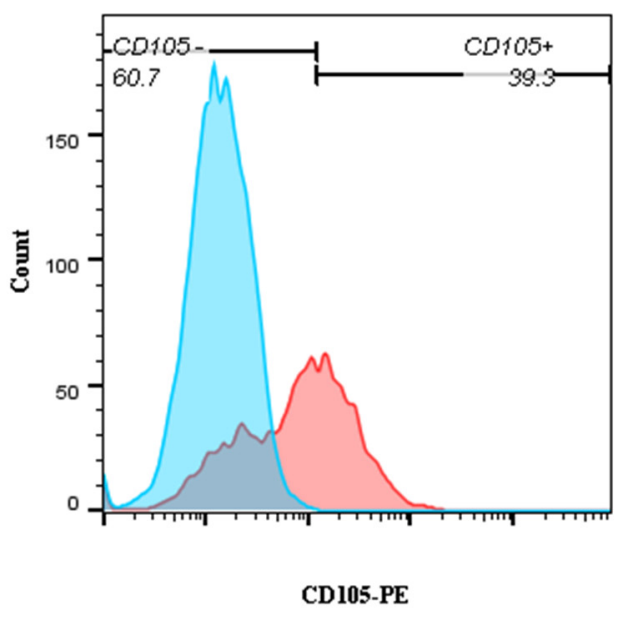

Figure 9. The results of flow cytometry histograms again to express CD73, CD90, CD105, CD34 markers of OMSCs after 21 day of differentiation
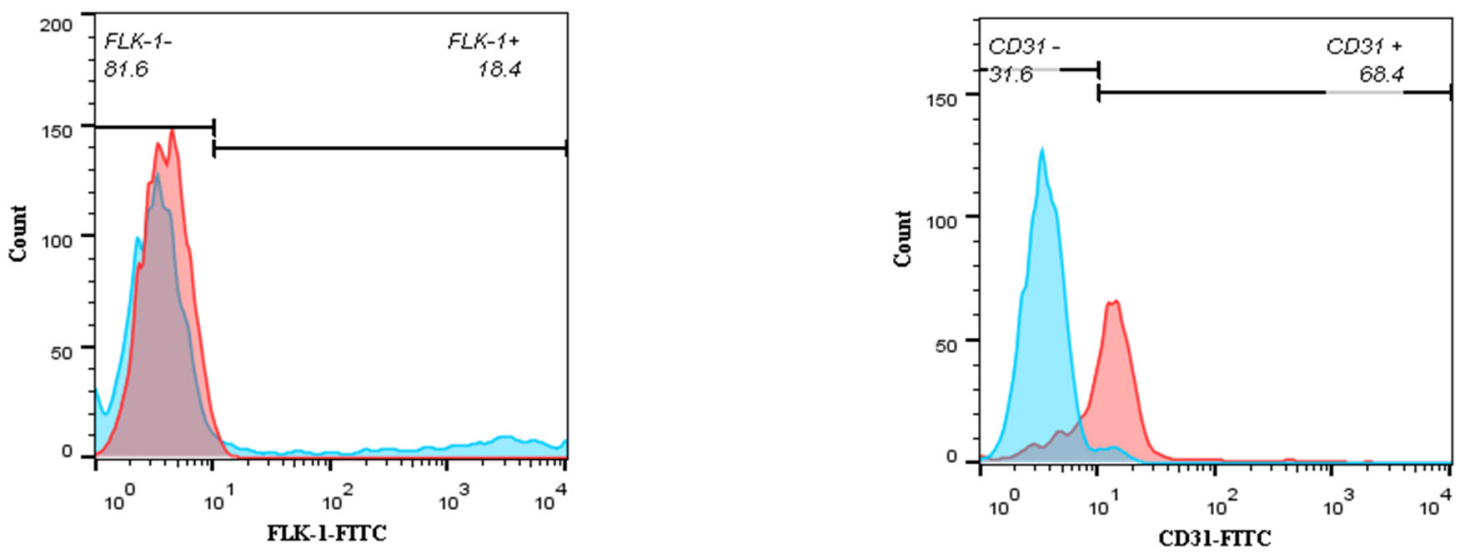

Figure 10. Flow cytometric analysis with cell surface markers CD31 and FLK1. Flow cytometric. Analysis showed expression of CD31 and FLK1 on OMSCs. These findings indicated that, OMSCs differentiated into endothelial progenitor cells 
In the charts, the expression of mesenchymal markers of the first test, significant changes had occurred. CD73 and CD90 expressions of CD105 were reduced to $5.8 \%$ and the increase in CD34 expression was observed. So we are looking for the cell that expressions CD34. Investigation, led us to the endothelial cell. The cells morphology also ptoved exacerbated this hypothesis. The expression of CD31 and FLK1 other key markers of early expression in epithelial cells are tested with flow cytometry test. The main markers for the detection of epithelial cells, CD31, CD34 and FLK1 showed a positive expression (Figure10) (Pusztaszeri et al., 2006).

\section{Discussion}

In this reserch work, our goal was to find that MSCs in the presence of a source of hormones and growth factors such as placenta extract what caused this situation and which factors were involved? MSCs are pluripotent progenitors that can differentiate into a variety of cell types. Vascular endothelial growth factor (VEGF) is one of the major factors of initiating and regulating angiogenesis. It has been reported that VEGF can induce OMSCs differentiated into endothelial cells (ECs) (Meirelles et al., 2009). In our tests, VEGF expressed in high level and in different ways. We have to find the reason for the differentiation of cells, need to get a few spots that we continue to discuss it. The first source were MSCs them cells. MSCs during tissue repair, MSCs respond to stressactively or injury in a manner that is look like to how the adaptive and innate immune system cells respond to pathogen exposure or apoptosis. When supplied exogenously, MSCs in Faced with injury inflamed or broken blood vessels, shown an intricate array of soluble mediators generated in a specific manner, the requirement of the environment. These products can promote angiogenesis, regeneration, remodeling, immune cell activation or suppression, and cellular recruitment. At the same time the MSCs can also actively participate in bactericidal activity. MSCs to repair tissues, can either distal or endocrine and local paracrine their effects have included measures such as: the development of angiogenesis by vascular endothelial growth factor (VEGF), insulin-like growth factor 1 (IGF-1) protein, monocyte chemoatractant protein1 (MCP1), basic fibroblast growth factor (bFGF) and interleukin 6 (IL6) (Wang et al., 2013). For a damaged tissue, the presence of molecules needed to work to gether. Every one of them as a group and team work is essential. According to the (Figure 2) molecules such as bFGF VEGF, HGF, IGF-1, TGF_B, and so, Should cooperate by each other to successfully repairs the vessel and angiogenesis to be completed (Fernando et al., 2012). In our test environment, rich source of placenta extract and molecules that were released from their OMSCs, were provided all of molecules and hormones that OMSCs required to endothelialization. The second source, is placenta and placental trophoblast. The development of a placental vascular network is essential for the growth and maintenance of the developing embryo. Several factors are involved in this angiogenic process, including VEGF (vascular endothelial growth factor), PDGF (platelet-derived growth factor) and PAF (platelet-activating factor).VEGF induces angiogenesis and increases permeabilization of blood vessels. VEGF and its receptors are expressed in both the endometrium and in trophoblastic cells (Asahara et al., 1997). VEGF mRNA expression can be detected in the blastocyst, enabling the implanting embryo to induce angiogenesis at the implantation site by binding to endometrial receptors. In the beginning of the period of embryogenesis, VEGF expression is in high level to induce angiogenesis at the site of implantation and a connection to endometrial receptors are essential. By the development of the placenta, another angiogenic factors, namely, PIGF (placental growth factor) angiogenic factor and its expression is extremely high (Krüssel et al., 2003). The third source of VEGF is PIGF (placental growth factor) in placenta (placental extract). PDGF expression of trophoblast cells and its subset of families VEGF located. Platelet-derived growth factor (PDGF) is one of the numerous growth factors, or proteins that regulate cell growth and division. In particular, it plays a significant role in blood vessel formation (angiogenesis). PDGFs are mitogen during early developmental stages, driving the proliferation of undifferentiated mesenchyme and some progenitor populations. During later maturation stages, PDGF signaling has been implicated in tissue remodeling and cellular differentiation, and in inductive events involved in patterning and morphogenesis. In addition to driving mesenchymal proliferation (Hannink et al., 1989). Another point is the differentiation of endothelial cells why mesenchymal cells are not affected by other factors that were present in the placental extract, and why doesn't differentiate to other classes such as bone, fat or cartilage cells? They have a capacity for differentiation into osteoblasts, adipocytes, and chondroblasts in vitro For MSC definition adherence to plastic in standard culture conditions. Thus, for example, expressed BMP, show bone morphogenetic characteristics, Protein EGF, epidermal growth factor or FGF, fibroblast growth factor, which are regulatory factors. MEF, myogenic factor that will have the muscle cells, and expression of VEGF, vascular endothelial growth factor causes OMSCs to endothelial cells. But The PDGF, platelet-derived growth factor! This growth factor acts as an inhibitor to differentiate OMSCs into cartilage and bone cells. Thus it can be proved that in the presence of this growth factor in placenta extract, they never differentiate into bone and cartilage line (Vasileios et al., 2015). About the cell cycle, it must be said that there is a relationship interface between cell proliferation and differentiation that is critical issue in cell biology. We know what model of differentiated somatic cells, the cell cycle and differentiation phenomenon is connected to each 
other. G1 is the cell cycle phase that altered during development and differentiation and G1 lengthensconcomitant with differentiation. G1 is regulated by the Cyclin E and Cyclin D1; the cyclin-dependent kinases (CDK) CDK2 and CDK4; the cyclin-dependent kinase inhibitors (CKI) p27 and p21; and the retinoblastoma protein (pRb) (Akiyama et al., 1992). It is assumed that lengthened G1 phase can be used as an indicator of differentiation status of individual human embryonic stem cells. Therefore OMSCs are more easily obtainable in large quantities and so the placenta that has this amazing material can be used as powerful motivating factor for the differentiation of OMSCs that are capable of differentiation.

\section{Conclusion}

In general, the findings of this approach can be concluded that based on morphological changes and expression of specific markers of endothelial, MSCs derived from omentum, by treatment by placenta extract, differentiation of endothelial cells are formed. Since the purified OMSCs derived from omentum, low-dose induction can be considered a good texture. Purification of cells by various origins allows researchers and clinicians in treatment to have fewer problems, as well as the placenta having this amazing material can be used as the driving force in the differentiation of OMSCs cells capable of differentiation. Placenta due to its nature and rich materials, has great potential for medical and applications, treatments.

\section{Acknowledgment}

The authors have special thanks to the Science and Research Branch, Islamic Azad University, Tehran, Iran for their support of this work as a $\mathrm{PhD}$ dissertation.

\section{References}

Akiyama, T., Ohuchi, T., Sumida, S., Matsumoto, K., \& Toyoshima, K. (1992). Phosphorylation of the retinoblastoma protein by cdk2. Proceedings of the National Academy of Sciences, 89(17), 7900-7904.

Asahara, T., Murohara, T., Sullivan, A., Silver, M., van der Zee, R., Li, T., \& Isner, J. M. (1997). Isolation of putative progenitor endothelial cells for angiogenesis. Science, 275(5302), 964-966.

Athanassiades, A., \& Lala, P. K. (1998). Role of placenta prowth factor (PIGF) in human extravillous trophoblast proliferation, migration and invasiveness. Placenta, 19(7), 465-473.

Bunnell, B. A., Flaat, M., Gagliardi, C., Patel, B., \& Ripoll, C. (2008). Adipose-derived stem cells: isolation, expansion and differentiation. Methods, 45(2), 115-120.

Calder, A., Roth-Albin, I., Bhatia, S., Pilquil, C., Lee, J. H., Bhatia, M., ... \& Draper, J. S. (2012). Lengthened G1 phase indicates differentiation status in human embryonic stem cells. Stem cells and development, 22(2), 279-295.

Chen, K. S., Chen, W. S., Chen, H. Y., Lee, C. C., Kao, H. S., Chen, Y. C., \& Ting, M. K. (2012). Experience in primary culture of human peritoneal mesothelial cell. Chin J Physiol, 55, 274-283.

da Silva Meirelles, L., Fontes, A. M., Covas, D. T., \& Caplan, A. I. (2009). Mechanisms involved in the therapeutic properties of mesenchymal stem cells. Cytokine \& growth factor reviews, 20(5), 419-427.

Darvin Sandhaanam, S., Pathalam, G., Dorairaj, S., \& Savariar, V. (2013). Mesenchymal stem cells (MSC): Identification, Proliferation and Differentiation. A Review Article. P.G \& Research Department of Advanced Zoology and Biotechnology. Loyola College, Chennai, Tamil Nadu, India.

Dhanasekaran, M., Indumathi, S., Harikrishnan, R., Mishra, R., Lissa, R. P., Rajkumar, J. S., \& Sudarsanam, D. (2013). Human omentum fat-derived mesenchymal stem cells transdifferentiates into pancreatic islet-like cluster. Cell biochemistry and function, 31(7), 612-619.

DiMarino, A. M., Caplan, A. I., \& Bonfield, T. L. (2013). Mesenchymal stem cells in tissue repair. Frontiers in immunology, 4.

Dominici, M. L. B. K., Le Blanc, K., Mueller, I., Slaper-Cortenbach, I., Marini, F. C., Krause, D. S., ... \& Horwitz, E. M. (2006). Minimal criteria for defining multipotent mesenchymal stromal cells. The International Society for Cellular Therapy position statement. Cytotherapy, 8(4), 315-317.

Dyce, P. W., \& Li, J. (2006). From skin cells to ovarian follicles?. Cell Cycle, 5(13), 1371-1375.

Figueroa, F. E., Carrión, F., Villanueva, S., \& Khoury, M. (2012). Mesenchymal stem cell treatment for autoimmune diseases: a critical review. Biological research, 45(3), 269-277.

Hannink, M., \& Donoghue, D. J. (1989). Structure and function of platelet-derived growth factor (PDGF) and related proteins. Biochimica et Biophysica Acta (BBA)-Reviews on Cancer, 989(1), 1-10.

Hoeben, A., Landuyt, B., Highley, M. S., Wildiers, H., Van Oosterom, A. T., \& De Bruijn, E. A. (2004). Vascular endothelial growth factor and angiogenesis. Pharmacological reviews, 56(4), 549-580. 
Karantalis, V., \& Hare, J. M. (2015). Use of mesenchymal stem cells for therapy of cardiac disease. Circulation research, 116(8), 1413-1430.

Karaoz, E., Aksoy, A., Ayhan, S., Sarıboyacı, A. E., Kaymaz, F., \& Kasap, M. (2009). Characterization of mesenchymal stem cells from rat bone marrow: ultrastructural properties, differentiation potential and immunophenotypic markers. Histochemistry and cell biology, 132(5), 533.

Katzman, P. A., \& Doisy, E. A. (1932). Preparation of extracts of the anterior pituitary-like substance of urine of pregnancy. Journal of Biological Chemistry, 98(2), 739-754.

Kentaro, Y. (2001). Director of the Yoshida Clinic. Placenta Power: For Health and Beauty A useful guide for those seeking placenta-based remedies. Director of the Yoshida Clinic.

Klopp, A. H., Zhang, Y., Solley, T., Amaya-Manzanares, F., Marini, F., Andreeff, M., ... \& Lu, K. (2012). Omental adipose tissue-derived stromal cells promote vascularization and growth of endometrial tumors. Clinical cancer research, 18(3), 771-782.

Krüssel, J. S., Bielfeld, P., Polan, M. L., \& Simón, C. (2003). Regulation of embryonic implantation. European Journal of Obstetrics \& Gynecology and Reproductive Biology, 110, S2-S9.

Miyamoto, K., Hayashi, K., Suzuki, T., Ichihara, S., Yamada, T., Kano, Y., ... \& Ito, Y. (2004). Human placenta feeder layers support undifferentiated growth of primate embryonic stem cells. Stem cells, 22(4), 433-440.

Nikoozad, Z., Ghorbanian, M. T., \& Rezaei, A. (2014). Comparison of the liver function and hepatic specific genes expression in cultured mesenchymal stem cells and hepatocytes. Iranian journal of basic medical sciences, 17(1), 27.

Parolini, O., Soncini, M., Evangelista, M., \& Schmidt, D. (2009). Amniotic membrane and amniotic fluid-derived cells: potential tools for regenerative medicine?

Phil. Trans, R. Soc, B. (2011). The cell cycle. The Royal Society, 366, 3494-3497.

Pusztaszeri, M. P., Seelentag, W., \& Bosman, F. T. (2006). Immunohistochemical expression of endothelial markers CD31, CD34, von Willebrand factor, and Fli-1 in normal human tissues. Journal of Histochemistry \& Cytochemistry, 54(4), 385-395.

Ramos, T. L., Sánchez-Abarca, L. I., Muntión, S., Preciado, S., Puig, N., López-Ruano, G., ... \& Sánchez-Guijo, F. (2016). MSC surface markers (CD44, CD73, and CD90) can identify human MSC-derived extracellular vesicles by conventional flow cytometry. Cell Communication and Signaling, 14(1), 2.

Salehinejad, P., Alitheen, N. B., Ali, A. M., Omar, A. R., Mohit, M., Janzamin, E., ... \& Nematollahi-Mahani, S. N. (2012). Comparison of different methods for the isolation of mesenchymal stem cells from human umbilical cord Wharton's jelly. In Vitro Cellular \& Developmental Biology-Animal, 48(2), 75-83.

Sebaugh, J. L. (2011). Guidelines for accurate EC50/IC50 estimation. Pharmaceutical statistics, 10(2), 128-134.

Tandon, P. N. (2009). Transplantation and stem cell research in neurosciences: Where does India stand?. Neurology India, 57(6), 706.

Wang, N., Zhang, R., Wang, S. J., Zhang, C. L., Mao, L. B., Zhuang, C. Y., ... \& Zhang, T. C. (2013). Vascular endothelial growth factor stimulates endothelial differentiation from mesenchymal stem cells via Rho/myocardin-related transcription factor-A signaling pathway. The international journal of biochemistry \& cell biology, 45(7), 1447-1456.

Witowski, J., \& Jörres, A. (2006). Peritoneal cell culture: fibroblasts. Peritoneal Dialysis International, 26(3), 292-299.

Zhang, Y. I., Li, C., Jiang, X., Zhang, S., Wu, Y., Liu, B., ... \& Mao, N. (2004). Human placenta-derived mesenchymal progenitor cells support culture expansion of long-term culture-initiating cells from cord blood CD34+ cells. Experimental hematology, 32(7), 657-664.

Zhang, Y., Khan, D., Delling, J., \& Tobiasch, E. (2012). Mechanisms underlying the osteo-and adipo-differentiation of human mesenchymal stem cells. The Scientific World Journal, 2012.

\section{Copyrights}

Copyright for this article is retained by the author(s), with first publication rights granted to the journal.

This is an open-access article distributed under the terms and conditions of the Creative Commons Attribution license (http://creativecommons.org/licenses/by/4.0/). 